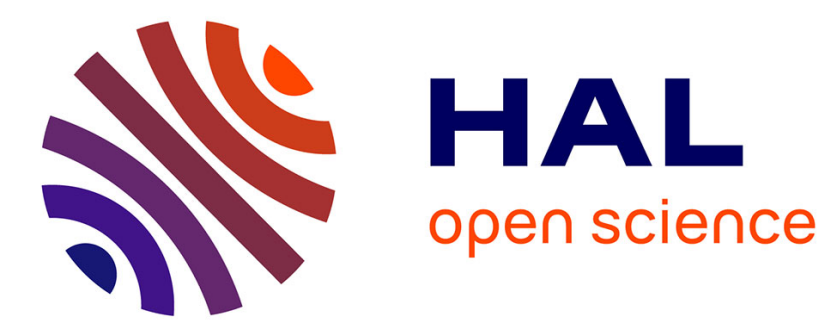

\title{
Routing game on the line: The case of multi-players
}

Abdelillah Karouit, Majed Haddad, Eitan Altman, Abdellatif El Matar

\section{To cite this version:}

Abdelillah Karouit, Majed Haddad, Eitan Altman, Abdellatif El Matar. Routing game on the line: The case of multi-players. UNet'2017 - Third International Symposium on Ubiquitous Networking, May 2017, Casablanca, Morocco. hal-01536349

\section{HAL Id: hal-01536349 \\ https://hal.inria.fr/hal-01536349}

Submitted on 11 Jun 2017

HAL is a multi-disciplinary open access archive for the deposit and dissemination of scientific research documents, whether they are published or not. The documents may come from teaching and research institutions in France or abroad, or from public or private research centers.
L'archive ouverte pluridisciplinaire HAL, est destinée au dépôt et à la diffusion de documents scientifiques de niveau recherche, publiés ou non, émanant des établissements d'enseignement et de recherche français ou étrangers, des laboratoires publics ou privés. 


\title{
Routing game on the line: The case of multi-players
}

\author{
Abdelillah Karouit ${ }^{1}$, Majed Haddad ${ }^{1}$, Eitan Altman $^{2}$, and Abdellatif El Matar ${ }^{3}$ \\ LIA/CERI University of Avignon, Agroparc, BP 1228, 84911, Avignon, France ${ }^{1}$ \\ akarouit@gmail.com,majed.haddad@univ-avignon.fr \\ INRIA Sophia Antipolis, 10 route des Lucioles, 06902 Sophia Antipolis, France ${ }^{2}$ \\ eitan.altman@inria.fr \\ L-IR2M, Faculty of Sciences and Techniques Hassan 1st, University, Settat, Morocco ${ }^{3}$ \\ moulay.elmater@gmail.com
}

\begin{abstract}
In this paper, we study a sequential routing game networks where $M$ users send traffic to a destination on a line. Each user arrives at time epoch with a given capacity. Then, he ships its demand over time on a shared resource. The state of a player evolves according to whether he decides to transmit or not. The decision of each user is thus spatiotemporal control. We provide explicit expression of the equilibrium of such systems and compare it to the global optimum case. In particular, we compute the price of anarchy of such scheme and identify a Braesstype paradox in the context of sequential routing game.
\end{abstract}

Keywords: Sequential routing game, Nash equilibrium, price of anarchy, Braess-type paradox.

\section{Introduction}

We consider in this paper a routing game problem in a network where non cooperative agents (to whom we refer as players) wish to establish a paths from source to destination in order to transport a fixed amount of total traffic. In the context of telecommunication networks, a given network is shared by users while routes are chosen by the players so as to minimize the delay. A similar setting has been studied in the context of road traffic networks, where each player can be viewed as a transportation company which is to ship a flow of vehicles.

A natural framework within this class of problems can be analyzed using the non-cooperative game theory. An appropriate solution concept in noncooperative game theory is Nash equilibrium. At the Nash equilibrium, no user can gain by unilaterally deviating from his own policy.

Non-cooperative routing has long been studied both in the framework of road traffic, as well as in the framework of telecommunication networks. Such frameworks allow to model the flow configuration that results in networks in which routing decisions are made in a non-cooperative and distributed manner between 
the users.

There can be finite or infinite number of users. In the case of a infinite players each player is assumed to be atomless. By atomless we mean that the impact of routing choices of a single player on the utilities of other players is negligible. The resulting flow configuration corresponds to the Wardrop equilibrium [15]. This concept, has long been studied in the context of road traffic where there is an infinite of players (drivers) [16]. In the telecommunication community, Orda, Rom and Shimkin [17] consider that the number of players is finite, where a player (typically corresponding to a service provider) takes the routing decisions for the whole class of users that it controls. It then decides on how to split the demand it controls between various possible routes. They establish existence and uniqueness of Nash equilibrium over large class of general cost functions. This approach also appeared in the road traffic literature (e.g. [18]), but was not much used there. Such a routing game may be handled by models similar to [19] in the special case of a topology of parallel links. An alternative class of routing games is the one in which a player has to route all the demand it controls through the same path. A special case of such framework is the "congestion games" introduced by Rosenthal in [20]. In [24], a load balancing network and Kameda type paradox has been studied, where losses occur on links in a way that may depend on the congestion. All the above works have been well studied in time-invariant networks in the last few years.

Our focus here is a spatio-temporal competitive routing where the network is shared by several users which each one having a non negligible cost. The demand has to be split not only over space but also over time (see Figure 1). As an example, assume that $M$ players have each its own demand which should be shipped within a $T$ days from a given source to a destination. Thus a player has to split its demand into that corresponding to each of the days of the week. At each day, the route corresponding to the daily demand of each player should be determined. Examples of such games in road traffic appear in [28].

A desired property for an equilibrium is efficiency, i.e., social optimality. It is well known that Nash equilibria in routing games are generally not efficient. In particular, this non efficiency can lead to paradoxical phenomena e.g., the Braess paradox where adding a link to the network could result in an increase of cost to all users. For specific examples of non efficient behavior and paradoxes we cite for road traffic [22] and [25].

Our starting point here is the work [21] in which the authors have already studied a routing game on a line where the decision of a user is spatio-temporal control. They addressed the case where only a single user arrives at time epoch. The game considered in [21] assumes that a single user ships its own traffic over a line. However, this assumption may not always be true. Indeed, several users 
can coexist and competitively need to ship their own resource over a line.

In this paper, we extend their game problem into a general problem, where multiple users arrives on line. In particular, we consider an extreme scenario in which all traffic that arrives at a node could be shipped at the next node over a line. This case is extreme since it is the most unbalanced one, in the sense that the traffic that a player sends through the direct path has only one hop to go. We show that even with this simple demand matrix, which is clearly biased in favor of choosing the direct path, we establish the counter-intuitive fact: "It is possible that not all players send their traffic through the direct path at equilibrium". Examples of such games in road traffic can be found in [22], [24], [23] and [25].

In rest of the paper, we assume that $M$ players have their own demands which should be shipped within a week from a given source to a destination. Thus each player has to split its demand into that corresponding to each of the days of the week. At each day, the route corresponding to the daily demand of each player should be determined.

In the next Section, we present the system model along with the assumptions considered within the paper. Next, we give explicit expressions of the Nash equilibrium and the global optimum, respectively in Section III and Section IV. Section $\mathrm{V}$ presents some performance results including price of anarchy and Braess-type paradox. We conclude the paper in Section VI.

\section{The model}

Let $G=(\mathcal{N}, \mathcal{L}, \mathcal{I}, \mathcal{P})$ be a network routing game with $\mathcal{N}$ the set of nodes and $\mathcal{L}$ the set of links, $\mathcal{I}$ is the set of classes (e.g. players), and $P=\left(s_{i}, d^{i}, \phi_{i}\right)$ is a set that characterizes class $i$ : $s^{i}$ is the source, $d^{i}$ is the destination and $\phi_{i}$ is the demand related to player $i$.

We describe the system with respect to the variables $x_{l}^{i}$ which are restricted by the non-negativity constraints for each link $l$ and player $i: x_{l}^{i} \geq 0$ and by the conservation constraints for each player $i$ and each node $v$ :

$$
r_{v}^{i}+\sum_{j \in \operatorname{In}(v)} x_{j}^{i}=\sum_{j \in \operatorname{Out}(v)} x_{j}^{i}
$$

where $r_{v}^{i}=\phi_{i}$ if $v$ is the source node for player $i, r_{v}^{i}=\phi_{i}$ if $v$ is its destination node, and $r_{v}^{i}=0$ otherwise; $\operatorname{In}(v)$ and $\operatorname{Out}(v)$ are respectively all ingoing and outgoing links of node $v$.

A player $i$ determines the routing decisions for all the traffic that corresponds to the corresponding class $i$. The cost of player $i$ is assumed to be additive over links

$$
J^{i}(\mathbf{x})=\sum_{l} J_{l}^{i}\left(\mathbf{x}_{l}\right)
$$

We shall assume that 
(i) $K_{l}^{i}:=\frac{\partial J_{l}^{i}(\mathbf{x})}{\partial x_{l}^{i}}$ exist and are continuous in $x_{l}^{i}$ (for all $i$ and $l$ ),

(ii) $J_{l}^{i}$ are convex in $x_{l}^{i}$ (for all $i$ and $l$ ),

We shall also make the following assumptions for each link $l$ and player $i$ :

A1: $J_{l}^{i}$ depends on $\mathbf{x}_{l}$ only through the total flow $x_{l}$ and the flow of $x_{l}^{i}$ of player $i$ over the link.

A2: $J_{l}^{i}$ is increasing in both arguments.

A3: Whenever $J_{l}^{i}$ is finite, $K_{l}^{i}\left(x_{l}, x_{l}^{i}\right)$ is strictly increasing in both arguments.

We further restrict the cost to satisfy the following:

B1: For each link $l$ there is a nonnegative cost density $T_{l}\left(x_{l}\right) . T_{l}$ is a function of the total flow through the link and $J_{l}^{i}=x_{l}^{i} T_{l}\left(x_{l}\right)$.

B2: $T_{l}$ is positive, strictly increasing and convex, and is continuously differentiable.

The Lagrangian with respect to the constraints on the conservation of flow is

$$
L_{i}(\mathbf{x}, \lambda)=\sum_{l \in \mathcal{L}} J_{l}^{i}\left(x_{l}, x\right)+\sum_{v \in \mathcal{N}} \lambda_{v}^{i}\left(r_{v}^{i}+\sum_{j \in \operatorname{In}(v)} x_{j}^{i}-\sum_{j \in O u t(v)} x_{j}^{i}\right),
$$

for each player $i$. Thus a vector $\mathbf{x}$ with nonnegative components satisfying (1) for all $i$ and $v$ is an equilibrium if and only if the following Karush-Kuhn-Tucker (KKT) condition holds. Below we shall use $u v$ to denote the link defined by node pair $u, v$. There exist Lagrange multipliers $\lambda_{u}^{i}$ for all nodes $u$ and all players, $i$, such that for each pair of nodes $u, v$ connected by a directed link $(u, v)$,

$$
K_{u v}^{i}\left(x_{u v}^{i}, x_{u v}\right) \geq \lambda_{u}^{i}-\lambda_{v}^{i},
$$

with equality if $x_{u v}>0$.

Assume cost structure $B$. Then, the Lagrangian is given by

$$
\begin{aligned}
& L_{i}(\mathbf{x}, \lambda)=\sum_{l \in \mathcal{L}}\left[T_{l}\left(x_{l}\right)+x_{l}^{i} \frac{\partial T_{l}\left(x_{l}\right)}{\partial x_{l}}\right]+ \\
& \quad \sum_{v \in \mathcal{N}} \lambda_{v}^{i}\left(r_{v}^{i}+\sum_{j \in \operatorname{In}(v)} x_{j}^{i}-\sum_{j \in \operatorname{Out}(v)} x_{j}^{i}\right),
\end{aligned}
$$

for each player $i$. Equation (4) can be written as

$$
T_{u v}\left(x_{u v}\right)+x_{u v}^{i} \frac{\partial T_{u v}\left(x_{u v}\right)}{\partial x_{u v}} \geq \lambda_{u}^{i}-\lambda_{v}^{i} .
$$

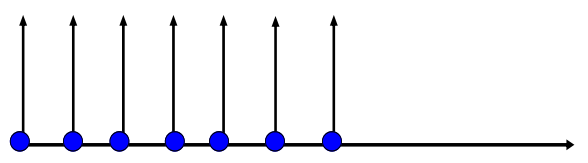

Fig. 1. Competitive routing on the line. 


\section{The transient equilibrium for multi-user sequential game}

Consider a sequential game: Assume at each day $i$, there are $M$ new players that arrive. Player $i$ comes with a demand of $\phi_{i}$ each competing for the link. This demand has to be shipped within 2 days. The decision of each player influences the cost of the others. This is a scheduling game: how much should player $i$ send upon arrival and how much should it delay to the next day? If the game is already on for a long time, we can expect to have a symmetric solution (this will be made exact below). This means that at each day, the same amount will be sent. And since each day an amount of $\phi_{i}$ arrives, then the amount that will have to leave each day is also $\phi_{i}$. An optimal solution will therefore be to send immediately all the arriving demand. We shall see however that at equilibrium, each player sends some of its traffic in the next day. We solve this problem by viewing it as an equivalent routing problem. The cost per unit of packet sent each day is $f(x)$. The cost for waiting another day is $d$.

Formally, let the demand of each player $k=1,2, \cdots, M$ arriving at day $i$ be $\phi_{i, k}>0$ that has to be shipped to destination within a period of 2 days. Let $x_{j}^{i, k}$ denotes the amount of flow sent by user $k$ on day $j$ knowing that user $k$ arrived at day $i$. The total flow on day $i$ is then denoted as $x_{i}=\sum_{k=1}^{M} x_{i}^{i, k}+x_{i}^{i-1, k}$. Let the vector $\mathbf{x}^{i, k}=\left(x_{i}^{i, k}, x_{i+1}^{i, k}\right)$ denote the amount of flow sent by user $k$ arriving at day $i$. The vector $\mathbf{x}^{i, k}$ is said to be feasible if $x_{i}^{i, k}+x_{i+1}^{i, k}=\phi_{i, k}$. For a given flow configuration of users $\left(\mathbf{x}^{i, 1}, \mathbf{x}^{i, 2}, \cdots, \mathbf{x}^{i, M}\right)$, user $k$ pays a congestion cost of $f\left(x_{i}\right)$ and delay cost of $d$ per unit of its flow on day $i$. The objective of user $l$ arriving at day $i$ is to minimize his cost given by

$$
J^{i, l}=x_{i}^{i, l} f\left(x_{i}\right)+x_{i+1}^{i, l}\left(f\left(x_{i+1}\right)+d\right)
$$

By differentiating the cost function with respect to $x_{i}^{i, l}$ and setting the derivative equal to zero, we get:

$$
x_{i}^{i, l}=\frac{d+f\left(x_{i+1}\right)-f\left(x_{i}\right)+\phi_{i, l} g\left(x_{i+1}\right)}{g\left(x_{i}\right)+g\left(x_{i+1}\right)}
$$

so that

$$
x_{i+1}^{i, l}=\phi_{i, l}-x_{i}^{i, l}=\frac{-d-f\left(x_{i+1}\right)+f\left(x_{i}\right)+\phi_{i, l} g\left(x_{i}\right)}{g\left(x_{i}\right)+g\left(x_{i+1}\right)}
$$

and also

$$
x_{i+1}^{i+1, l}=\frac{d+f\left(x_{i+2}\right)-f\left(x_{i+1}\right)+\phi_{i, l} g\left(x_{i+2}\right)}{g\left(x_{i+1}\right)+g\left(x_{i+2}\right)}
$$

Taking the sum, we obtain

$x_{i+1}=\sum_{k=1}^{M} \frac{d+f\left(x_{i+2}\right)-f\left(x_{i+1}\right)+\phi_{i, k} g\left(x_{i+2}\right)}{g\left(x_{i+1}\right)+g\left(x_{i+2}\right)}+\frac{-d-f\left(x_{i+1}\right)+f\left(x_{i}\right)+\phi_{i, k} g\left(x_{i}\right)}{g\left(x_{i}\right)+g\left(x_{i+1}\right)}$ 


\subsection{The steady state}

In the steady state, we have $x_{i}=\sum_{k=1}^{M} \phi_{i, k}, \forall i$. Hence, for every user $l$ we have from equation (8)

$$
x_{i}^{i, l}=\frac{\phi_{i, l}}{2}+\frac{d}{2 g\left(\sum_{k=1}^{M} \phi_{i, k}\right)}
$$

\subsection{The case of linear cost}

Similarly to the previous sections, let $f(x)=a x$. Then, equation (8) gives

$$
-x_{i+2}+\left(\frac{2}{M}+2\right) x_{i+1}-x_{i}=\left(\frac{2}{M}\right) \sum_{k=1}^{M} \phi_{k}
$$

The solution of this difference equation has the form

$$
x_{i}=c_{1}\left(r_{1}\right)^{i}+c_{2}\left(r_{2}\right)^{i}+\sum_{k=1}^{M} \phi_{k}
$$

where $r_{1}$ and $r_{2}$ are the solution of the characteristic equation

$$
-r^{2}+\left(\frac{2}{M}+2\right) r-1=0
$$

They are thus given by

$$
r_{1,2}=1+\frac{1}{M}(1 \pm \sqrt{1+2 M})
$$

Similarly, using the condition $x_{0}=0$ we conclude that

$$
x_{i}=\sum_{k=1}^{M} \phi_{k}\left[1-\left(1+\frac{1}{M}(1-\sqrt{1+2 M})\right)^{i}\right]
$$

\section{The global optimum for a semi infinite line}

Let us now compute the global cost for the multi-user case at day $i$.

$$
J^{i}(\mathbf{x})=\sum_{i=1}^{M} J^{i, l}(\mathbf{x})
$$

Deriving the sum with respect to $x_{i}^{i, l}$, we have

$$
\frac{\partial J^{l}(\mathbf{x})}{\partial x_{i}^{i, l}}=f\left(x_{i}\right)+x_{i}^{i, l} g\left(x_{i}\right)-\left(\phi_{i, l}-x_{i}^{i, l}\right) g\left(x_{i+1}\right)-f\left(x_{i+1}\right)-d+\left(\phi_{i-1, l}-x_{i-1}^{i-1, l}\right) g\left(x_{i}\right)-x_{i+1}^{i+1, l} g\left(x_{i+1}\right)
$$


Equating the above equation to zero gives

$$
x_{i}^{i, l}=\frac{d+f\left(x_{i+1}\right)-f\left(x_{i}\right)+\phi_{i, l} g\left(x_{i+1}\right)}{g\left(x_{i}\right)+g\left(x_{i+1}\right)}-\frac{\phi_{i-1, l} g\left(x_{i}\right)+x_{i+1}^{i+1, l} g\left(x_{i+1}\right)+x_{i-1}^{i-1, l} g\left(x_{i}\right)}{g\left(x_{i}\right)+g\left(x_{i+1}\right)}
$$

so that

$$
\phi_{i}-x_{i}^{i, l}=\frac{-d-f\left(x_{i+1}\right)+f\left(x_{i}\right)+\phi_{i, l} g\left(x_{i}\right)}{g\left(x_{i}\right)+g\left(x_{i+1}\right)}+\frac{\phi_{i-1, l} g\left(x_{i}\right)-x_{i+1}^{i+1, l} g\left(x_{i+1}\right)-x_{i-1}^{i-1, l} g\left(x_{i}\right)}{g\left(x_{i}\right)+g\left(x_{i+1}\right)}
$$

and also

$x_{i+1}^{i+1}=\frac{d+f\left(x_{i+2}\right)-f\left(x_{i+1}\right)+\phi_{i+1, l} g\left(x_{i+2}\right)}{g\left(x_{i+1}\right)+g\left(x_{i+2}\right)}-\frac{\phi_{i, l} g\left(x_{i+1}\right)+x_{i+2}^{i+2, l} g\left(x_{i+2}\right)+x_{i}^{i, l} g\left(x_{i+1}\right)}{g\left(x_{i+1}\right)+g\left(x_{i+2}\right)}$

Taking the sum, we obtain

$$
\begin{aligned}
x_{i+1}= & \sum_{k=1}^{M} \frac{-d-f\left(x_{i+1}\right)+f\left(x_{i}\right)+\phi_{i, l} g\left(x_{i}\right)}{g\left(x_{i}\right)+g\left(x_{i+1}\right)}+\frac{\phi_{i-1, l} g\left(x_{i}\right)-x_{i+1}^{i+1, l} g\left(x_{i+1}\right)-x_{i-1}^{i-1, l} g\left(x_{i}\right)}{g\left(x_{i}\right)+g\left(x_{i+1}\right)}+ \\
& \sum_{k=1}^{M} \frac{d+f\left(x_{i+2}\right)-f\left(x_{i+1}\right)+\phi_{i+1, l} g\left(x_{i+2}\right)}{g\left(x_{i+1}\right)+g\left(x_{i+2}\right)}-\frac{\phi_{i, l} g\left(x_{i+1}\right)+x_{i+2}^{i+2, l} g\left(x_{i+2}\right)+x_{i}^{i, l} g\left(x_{i+1}\right)}{g\left(x_{i+1}\right)+g\left(x_{i+2}\right)}
\end{aligned}
$$

\subsection{The case of linear cost}

Let $f(x)=a x$ and assume that $\phi_{i, k}=\phi_{k}, \forall i, k$ does not depend on $i$. Then

$$
-\frac{M}{2} x_{i+2}+(M+1) x_{i+1}-\frac{M}{2} x_{i}=\sum_{k=1}^{M} \phi_{k}+\theta
$$

where $\theta=\frac{1}{2} \sum_{k=1}^{M} x_{i+2}^{i+2, k}-x_{i+1}^{i+1, k}+x_{i}^{i, k}-x_{i-1}^{i-1, k}$. Noting $a_{i}=\sum_{k=1}^{M} x_{i}^{i, k}$, we have

$$
\begin{gathered}
x_{i+1}=\sum_{k=1}^{M} \phi_{k}-a_{i}+a_{i+1} \\
x_{i+2}=\sum_{k=1}^{M} \phi_{k}-a_{i+1}+a_{i+2}
\end{gathered}
$$

From (25) and (26), we obtain

$$
\begin{aligned}
\theta & =\frac{1}{2}\left[a_{i+2}-a_{i+1}+-a_{i}-a_{i+1}\right] \\
& =\frac{1}{2}\left[x_{i+2}-x_{i+1}\right]
\end{aligned}
$$

We then have the following recursive function

$$
-(M+1) x_{i+2}+(2 M+3) x_{i+1}-M x_{i}=2 \sum_{k=1}^{M} \phi_{k}
$$


The solution of this difference equation has the form

$$
x_{i}=c_{1}\left(r_{1}\right)^{i}+c_{2}\left(r_{2}\right)^{i}+\sum_{k=1}^{M} \phi_{k}
$$

where $r_{1}$ and $r_{2}$ are the solution of the characteristic equation $-(M+1) r^{2}+$ $(2 M+3) r-M=0$. They are thus given by

$$
r_{1,2}=1+\frac{1 \pm \sqrt{9+8 M}}{2(M+1)}
$$

Using the condition $x_{0}=0$, we conclude that, for every user $l$, we have

$$
x_{i}=\sum_{k=1}^{M} \phi_{k}\left[1-\left(1+\frac{1-\sqrt{9+8 M}}{2(M+1)}\right)^{i}\right]
$$

\section{Performance Analysis}

\subsection{Price of Anarchy}

At the steady state, the cost for player $i$ is

$$
\begin{aligned}
J^{i, l}(d) & =x_{i}^{i} f\left(\sum_{k=1}^{M} \phi_{i, k}\right)+x_{i+1}^{i}\left(f\left(\sum_{k=1}^{M} \phi_{i, k}\right)+d\right) \\
& =\left(x_{i}^{i}+x_{i+1}^{i}\right) f\left(\sum_{k=1}^{M} \phi_{i, k}\right)+d x_{i+1}^{i} \\
& =\phi_{i, l} f\left(\sum_{k=1}^{M} \phi_{i, k}\right)+d\left(\frac{\phi_{i, l}}{2}-\frac{d}{2 g\left(\sum_{k=1}^{M} \phi_{i, k}\right)}\right)
\end{aligned}
$$

Since $x_{i+1}^{i} \geq 0$, we have $d \leq \phi_{i, l} g\left(\sum_{k=1}^{M} \phi_{i, k}\right) . J^{i, l}(d)$ is concave in $d$ with a maximum at $d=\frac{\phi i, l g\left(\sum_{k=1}^{M} \phi_{i, k}\right)}{2}$. Interestingly, we observe that at $d=\phi_{i, l} g\left(\sum_{k=1}^{M} \phi_{i, k}\right)$, the cost function $J^{i, l}(d)$ is minimized and equal to $\phi_{i, l} f\left(\sum_{k=1}^{M} \phi_{k}\right)$ which is equal to the cost at $d=0$.

The price of anarchy $(\mathrm{PoA})$ is then given by

$$
\begin{aligned}
P o A & =\frac{J^{i, l}(d)}{\phi_{i, l} f\left(\sum_{k=1}^{M} \phi_{i, k}\right)} \\
& =1+\frac{d\left(\frac{\phi_{i, l}}{2}-\frac{d}{2 g\left(\sum_{k=1}^{M} \phi_{i, k}\right)}\right)}{\phi_{i, l} f\left(\sum_{k=1}^{M} \phi_{i, k}\right)}
\end{aligned}
$$

For the numerical results we set the value of $\phi_{i, l}$ to 1 and $a=1$.

Notice that the PoA is equal to 1 for $d=0$ and $d=\phi_{i, l} g\left(\sum_{k=1}^{M} \phi_{i, k}\right)$. We depict in Figure 2 the price of anarchy as function of delay for different number of users. As already said, we notice that the PoA is equal to 1 for $d=0$, and $d=1$. We also remark that the PoA increases as the number of users increases which leads to the interaction of users and hence induces more cost to ship their demand over the line. 


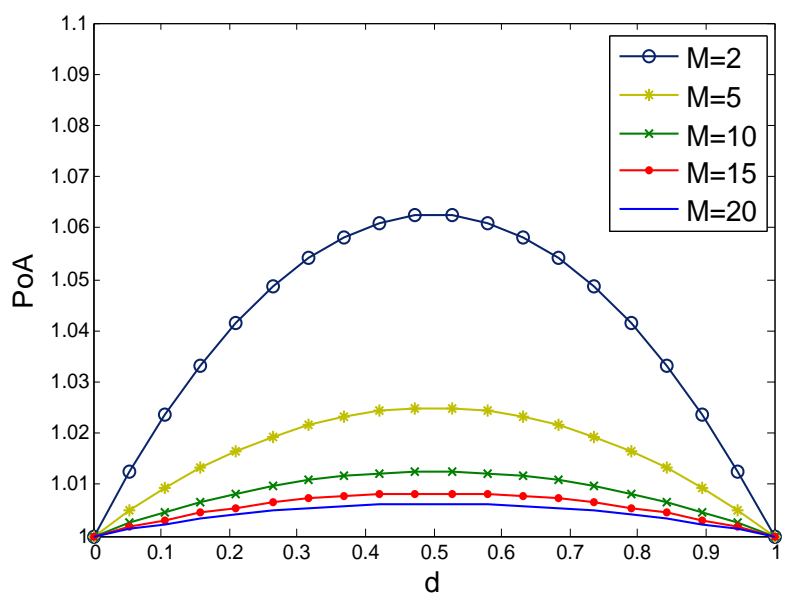

Fig. 2. Price of anarchy as function of cost

\subsection{Braess-type paradox}

At the steady state, the cost for player $l$ at day $i$ is:

$$
\begin{aligned}
J^{i, l}(d) & =x_{i}^{i} f\left(\sum_{k=1}^{M} \phi_{i, k}\right)+x_{i+1}^{i}\left(f\left(\sum_{k=1}^{M} \phi_{i, k}\right)+d\right) \\
& =\left(x_{i}^{i}+x_{i+1}^{i}\right) f\left(\sum_{k=1}^{M} \phi_{i, k}\right)+d x_{i+1}^{i} \\
& =\phi_{i, l} f\left(\sum_{k=1}^{M} \phi_{i, k}\right)+d\left(\frac{\phi_{i, l}}{2}-\frac{d}{2 g\left(\sum_{k=1}^{M} \phi_{i, k}\right)}\right) .
\end{aligned}
$$

It is clear that the cost for player $i$ is non-negative for

$$
d \in\left[\frac{\phi_{i, l} g\left(\sum_{k=1}^{M} \phi_{i, k}\right.}{2}, \phi_{i, l} g\left(\sum_{k=1}^{M} \phi_{i, k}\right)\right] .
$$

In this region, $J^{i, l}$ decreases as $d$ increases, which is a Braess type paradox (see Figure 3). This paradox was obtained in the context of a load balancing network in [25]. We depict in Figure 4 the the variation of the Nash equilibrium in Equation (17) and the global optimum in Equation (31) over the horizon for $\phi_{k}=1 ; \forall k=1, \ldots, M$. It is clearly illustrated that the Nash equilibrium over a semi infinite line converges to the global optimum from day $i=4$.

\section{Conclusion}

We have studied a sequential routing game on a line with several users, where each player comes with a fixed demand competing for the link and had to dispatch all its demand over a period of two days. Under some assumptions, we 


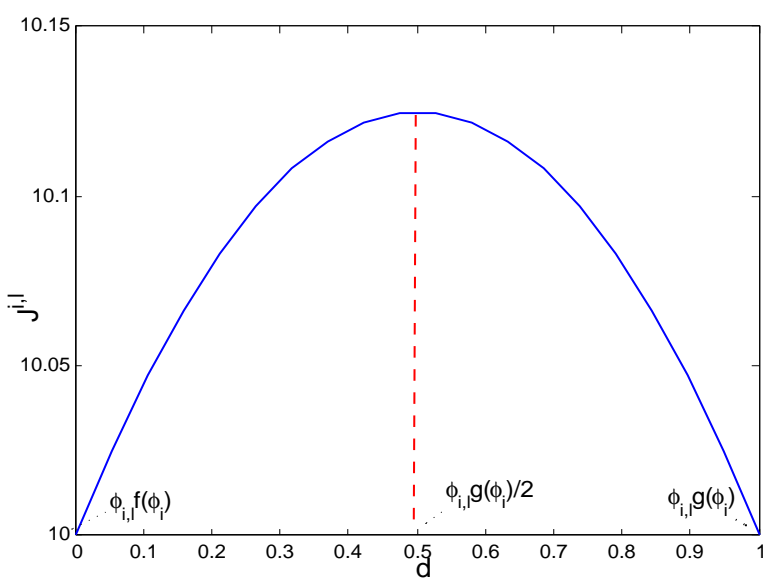

Fig. 3. The Braess-type paradox for the sequential game.

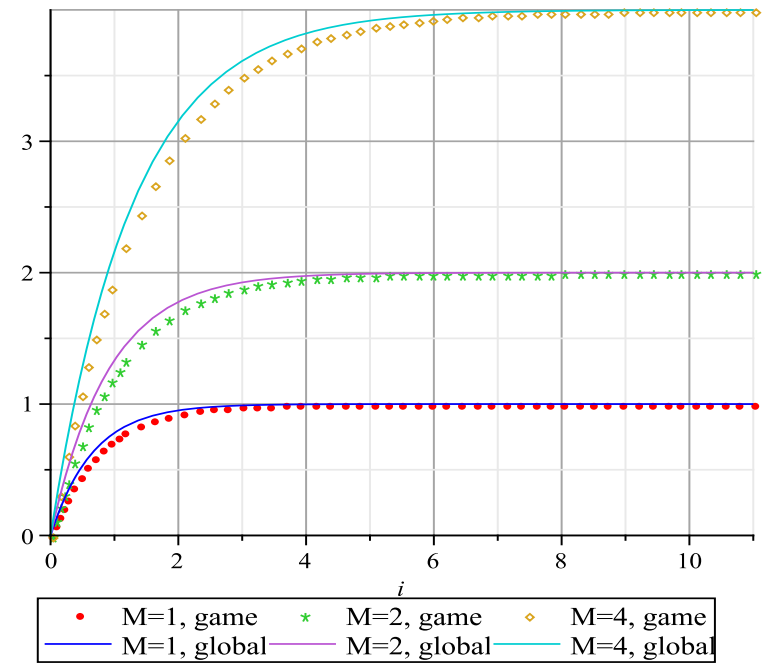

Fig. 4. Dynamics of the total flow sent over the horizon where $\phi_{k}=1 \forall k=1, \ldots, M$.

have obtained the explicit solutions for the Nash equilibrium and the global optimum and derive the price of anarchy. Finally, we have showed that under a semi infinite line, the Nash equilibrium converges to the global optimum and, have identified a Braess-type paradox behavior in the context of sequential games. 


\section{References}

1. A. Haurie and P. Marcotte. "On the relationship between Nash-Cournot and Wardrop equilibria". Networks, 15:295-308, 1985.

2. A. Orda, R. Rom and N. Shimkin, "Competitive routing in multi-user environments," IEEE/ACM Trans. on Networking, 510-521, 1993.

3. M. Patriksson, The traffic assignment problem: Models and methods, VSP, Utrecht, 1994.

4. R. W. Rosenthal, "A class of games possessing pure strategy Nash equilibria", Int. J. Game Theory, 2:65-67, 1973.

5. J. G. Wardrop. Some theoretical aspects of road traffic research communication networks. Proc. Inst. Civ. Eng., Part 2, 1:325-378, 1952.

6. K. Park, M. Sitharam, and S. Chen. "Quality of service provision in noncooperative networks with diverse user requirements", Decis. Support Syst., vol. 28, no.1-2, pp. 101-122, 2000.

7. U. Ayesta, O. Brun, B.J. Prabhu, "Price of Anarchy in Non-Cooperative Load Balancing", Proceedings of IEEE INFOCOM, San Diego, Mar. 2010.

8. R. Cominetti, J. R. Correa, N. E. Stier-Moses, "The Impact of Oligopolistic Competition in Networks", Operation Reserach,INFORMS, Vol. 57, No. 6, pp. 1421-1437, 2009.

9. D. Monderer, L. S. Shapley, "Potential Games", Games and Economic Behavior, 14, pp. 124-143, 1996.

10. M. K. Hanawal, E. Altman, R. El-Azouzi and B.J. Prabhu, "Spatio-temporal control for Dynamic Routing Games", Proceedings of Game Theory for Networks (GameNets), Shanghai, China, April 2011.

11. E. Altman, K. Avrachenkov, N. Bonneau, M. Debbah, R. El-Azouzi and D. Sadoc Menasche, "Constrained Cost-Coupled Stochastic Games with Independent State Processes" Operations Research Letters, Vol 36, pp 160-164, 2008.

12. E. Altman, T. Basar, T. Jimenez and N. Shimkin , "Competitive routing in networks with polynomial cost" , IEEE Trans. on Automatic Control, Vol 47, pp. 92-96, Jan. 2002.

13. N Nissan, T Roughgarden, E. Tardos, V.V. Vazirani, Algorithmic Game Theory, Cambrige University Press, 2009.

14. H. Kameda and O. Pourtallier, "Paradoxes in distributed decisions on optimal load balancing for networks of homogeneous computers", Journal of the ACM, Vol.49, No.3, pp.407-433, 2002.

15. J. G. Wardrop. Some theoretical aspects of road traffic research communication networks. Proc. Inst. Civ. Eng., Part 2, 1:325-378, 1952.

16. M. Patriksson, The traffic assignment problem: Models and methods, VSP, Utrecht, 1994.

17. A. Orda, R. Rom and N. Shimkin, "Competitive routing in multi-user environments," IEEE/ACM Trans. on Networking, 510-521, 1993.

18. A. Haurie and P. Marcotte. "On the relationship between Nash-Cournot and Wardrop equilibria". Networks, 15:295-308, 1985.

19. K. Park, M. Sitharam, and S. Chen. "Quality of service provision in noncooperative networks with diverse user requirements", Decis. Support Syst., vol. 28, no.1-2, pp. 101-122, 2000.

20. R. W. Rosenthal, "A class of games possessing pure strategy Nash equilibria", Int. J. Game Theory, 2:65-67, 1973. 
21. M. Haddad, E. Altman and J. Gaillard, "Sequential routing game on the line: Transmit or relay?" Poceeding of Communications and Information Technology (ICCIT), June 2012,

22. M. K. Hanawal, E. Altman, R. El-Azouzi and B.J. Prabhu, "Spatio-temporal control for Dynamic Routing Games", Proceedings of Game Theory for Networks (GameNets), Shanghai, China, April 2011.

23. E. Altman, T. Basar, T. Jimenez and N. Shimkin , "Competitive routing in networks with polynomial cost" , IEEE Trans. on Automatic Control, Vol 47, pp. 92-96, Jan. 2002.

24. Eitan Altman, Joy Kuri and Rachid El-Azouzi, "A routing game in networks with lossy links", 7th International Conference on NETwork Games COntrol and OPtimization (NETGCOOP), Oct 2014, Trento, Italy.

25. H. Kameda and O. Pourtallier, "Paradoxes in distributed decisions on optimal load balancing for networks of homogeneous computers", Journal of the ACM, Vol.49, No.3, pp.407-433, 2002.

26. D. Braess, "ber ein Paradoxon aus der Verkehrsplanung", Unternehmensforschung, No. 12 , pp. 258-268, 1968.

27. T. Roughgarden, "The Price of Anarchy", MIT Press, Cambridge, MA, 2005.

28. B.W.Wie, T. Friesz, and R. Tobin, ?Dynamic user optimal traffic assignment on congested multidestination networks,? Transportation Research, vol. 24B, no. 6, pp. 431?442, 1990. 